\title{
Long non-coding RNA RP11-84C13.1 promotes osteogenic differentiation of bone mesenchymal stem cells and alleviates osteoporosis progression via the miR-23b-3p/RUNX2 axis
}

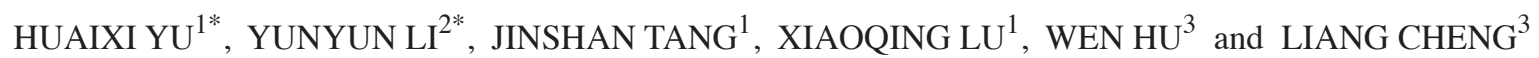 \\ Departments of ${ }^{1}$ Orthopaedic Surgery, ${ }^{2}$ Information Statistics Center and ${ }^{3}$ Endocrinology, Huai'an Second People's Hospital, \\ The Affiliated Huai'an Hospital of Xuzhou Medical University, Huai'an, Jiangsu 223000, P.R. China
}

Received December 26, 2020; Accepted August 17, 2021

DOI: $10.3892 /$ etm.2021.10775

\begin{abstract}
The objective of the present study was to determine the role of RP11-84C13.1 in osteoporosis (OP) and its molecular mechanism. First, clinical samples were collected from OP patients and normal control patients. Human bone marrow stromal cells (hBMSCs) were extracted from femoral head tissues. Runt-related transcription factor 2 (RUNX2) and RP11-84C13.1 serum levels were assessed by reverse transcription-quantitative (RT-q)PCR. Following transfection of pcDNA-RP11-84C13.1, si-RP11-84C13.1, microRNA (miRNA)-23b-3p mimic and miRNA-23b-3p inhibitor, the expression levels of RUNX2 and RP11-84C13.1 were determined by RT-qPCR. In addition, the osteogenic ability of hBMSCs was assessed by Alizarin Red staining. The binding of RP11-84C13.1 to miRNA-23b-3p and the binding of miRNA-23b-3p to RUNX2 was confirmed by dual-luciferase reporter gene assay. Long non-coding RNA (lncRNA) RP11-84C13.1 was significantly downregulated in the serum of OP patients. The osteogenic differentiation-related genes RUNX2 and RP11-84C13.1 were markedly upregulated in a time-dependent manner, while the miRNA-23b-3p level gradually decreased in hBMSCs with the prolongation of osteogenesis. RP11-84C13.1 knockdown inhibited the osteogenic differentiation of hBMSCs. Furthermore, RP11-84C13.1 regulated RUNX2 expression by targeting miRNA-23b-3p. Overexpression of miRNA-23b-3p partially reversed the promoting effect of RP11-84C13.1 on the osteogenesis of hBMSCs. In conclusion, lncRNA RP11-84C13.1 upregulated
\end{abstract}

Correspondence to: Dr Liang Cheng, Department of Endocrinology, Huai'an Second People's Hospital, The Affiliated Huai'an Hospital of Xuzhou Medical University, 60 Huaihai South Road, Huai'an, Jiangsu 223000, P.R. China

E-mail: chengliang0505@126.com

*Contributed equally

Key words: Long noncoding RNA RP11-84C13.1, microRNA-23b-3p, runt-related transcription factor 2, osteoporosis
RUNX2 by absorbing miRNA-23b-3p, and thus induced hBMSC osteogenesis to alleviate osteoporosis.

\section{Introduction}

Osteoporosis is an asymptomatic bone disease influenced by genetic, epigenetic, and environmental factors (1). It is characterized by loss of bone mass, increased bone fragility, and damaged bone tissue structure (2). With the improvement of higher socioeconomic status and the gradual aging of population, osteoporosis has become one of the most common and debilitating diseases, resulting in a huge economic burden on elderly health care, morbidity and mortality $(3,4)$. Early detection and intervention of osteoporosis risk can effectively delay the development of the disease and improve the quality of life of the patients (5). Hence, there remains an essential clinical need to identify molecular biomarkers and develop more effective interventions for osteoporosis.

Long noncoding RNAs (lncRNAs) are defined as transcripts longer than 200 nucleotides in length, and they usually have no protein-encoding capability $(6,7)$. Although they were previously considered to be transcriptional byproducts without any biological functions, emerging evidence has indicated that lncRNAs are involved in the mediated regulation of osteogenic differentiation and with osteoporosis pathogenesis at the transcriptional, post-transcriptional, and epigenetic levels (8-10). In a recent clinical study, lncRNA RP11-84C13.1 was markedly decreased in OP patients compared with normal healthy control patients (11). RP11-84C13.1 (ENSG00000271359) is a lncRNA on chromosome 4q22.1. There has been no in-depth study on RP11-84C13.1, to the best of our knowledge, except for the aforementioned study. However, the specific biological effects and molecular mechanisms of RP11-84C13.1 in osteoporosis progression remain unclear.

MicroRNAs (miRNAs) consist of a class of endogenous non-coding RNAs (ncRNAs) with a length of approximately 20-25 nucleotides that are recognized by complementary base pairing and guide the silencing complex to degrade targeted mRNA or suppress translation of targeted mRNA according to the degree of complementarity $(12,13)$. In addition, recent studies have demonstrated that IncRNAs can compete for miRNA-binding and act as 'sponges' or 'decoys' for miRNAs, 
thereby regulating the translation of target transcripts $(14,15)$. However, the potential clinical implications for miRNA-mediated IncRNA-associated competing endogenous RNA (ceRNA) in the pathogenesis of osteoporosis remain unknown.

In the present study, the expression pattern, biological function and underlying mechanism of action of newly discovered lncRNA RP11-84C13.1 in human bone marrow stromal cells (hBMSCs) were investigated. These results provided potential diagnostic and therapeutic targets for osteoporosis.

\section{Materials and methods}

Sample collection and patient data. The present study was approved by the Medical Ethics Committee of Huai'an Second People's Hospital (approval no. HEYLL201805; Huai'an, China) and all patients provided written informed consent. Blood samples were obtained from 23 osteoporosis patients (10 males and 13 females; age range, $45-78$ years) and 15 normal patients (6 males and 9 females; age range, 41-73 years) between January 2019 and December 2019. Blood samples were pre-cooled on ice for $30 \mathrm{~min}$. and then centrifuged at $2,500 \mathrm{x} \mathrm{g}$ at $4^{\circ} \mathrm{C}$ for $15 \mathrm{~min}$. The upper serum was harvested and stored at $-20^{\circ} \mathrm{C}$. Patients with cancer, rheumatoid arthritis, or metabolic diseases (e.g., diabetes, hyperthyroidism, hyperparathyroidism, severe kidney and liver disease) were excluded from the present study.

Isolation and culture of hBMSCs. Bone marrow samples were obtained from discarded femoral head tissue of individuals with or without osteoporosis during total hip arthroplasty (THA). Bone marrow was diluted (1:1) with $\alpha$-MEM (HyClone; Cytiva) and loaded over Percoll (Sigma-Aldrich; Merck KGaA) for density-gradient centrifugation. Mono-nucleated cells were obtained from the interface after centrifugation at $900 \mathrm{x} g$ at $4^{\circ} \mathrm{C}$ for $25 \mathrm{~min}$ and washed by phosphate-buffered saline (Gibco; Thermo Fisher Scientific, Inc.) twice. Then, cells were resuspended in $\alpha$-MEM containing 10\% FBS-HI (Gibco; Thermo Fisher Scientific, Inc.), $100 \mathrm{U} / \mathrm{ml}$ penicillin (HyClone; Cytiva) and $100 \mu \mathrm{g} / \mathrm{ml}$ streptomycin (HyClone; Cytiva) and seeded in T25 flasks (Corning, Inc.) at a density of $1 \times 10^{5}$ cells $/ \mathrm{cm}^{2}$ at $37^{\circ} \mathrm{C}$ and $5 \% \mathrm{CO}_{2}$. After $48 \mathrm{~h}$, medium change was conducted to remove non-adherent cells. The adherent cells were cultured for approximately 2 weeks until cell clones reached $\sim 75-85 \%$ confluence, then digested with $0.25 \%$ trypsin- $0.02 \%$ ethylenediaminetetraacetic acid (EDTA; Sigma-Aldrich; Merck KGaA) and finally sub-cultured at a density of $1 \times 10^{5} \mathrm{cells} / \mathrm{cm}^{2}$ in new T25 flasks. For the hBMSCs osteogenesis induction experiment, $10 \%$ FBS-HI was decreased to $1 \%$ FBS-HI to avoid possible proliferation and differentiation, and $50 \mu \mathrm{g} / \mathrm{ml}$ ascorbic acid, $10 \mathrm{mM} \beta$-glycerophosphate and $0.1 \mu \mathrm{g} / \mathrm{ml}$ dexamethasone (all from Sigma-Aldrich; Merck KGaA) were added. The osteogenic media was replaced every 3 days.

Cell transfection. When hBMSCs achieved 75-85\% confluency, the cells were transfected with Lipofectamine ${ }^{\mathrm{TM}} 2000$ (Invitrogen; Thermo Fisher Scientific, Inc.) according to the manufacturer's instructions. After transfection at $37^{\circ} \mathrm{C}$ for $6 \mathrm{~h}$, medium was replaced by $\alpha$-MEM containing $10 \%$ FBS-HI. pcDNA-NC, pcDNA-RP11-84C13.1, si-RP11-84C13.1\#1 (5'-GCCUCCGAGUAUCUCUACA-3'), si-RP11-84C13.1\#2
(5'-GCCAAAUCUAGUAGGUUUA-3'), si-RP11-84C13.1\#3 (5'-UCUACCUUGGGUCCUCAUU-3'), si-NC (5'-UUUGUA CUACACAAAAGUACUG-3'), si-RP11-84C13.1, NC mimics (5'-GGCUCUAGA AAAGCCUAUGC-3'), miR-23b-3p mimics (5'-AUCACAUUGCCAGGGAUUACCAC-3'), NC inhibitor (5'-CAGUACUUUUGUGUAGUACAAA-3') and miR-23b-3p inhibitor (5'-GUGGUAAUCCCUGGCAAUGUG AU-3') were purchased from Shanghai Genechem Co., Ltd. The concentration of transfected siRNA was $100 \mathrm{nM}$, and the plasmid concentration was $200 \mathrm{ng} / \mu \mathrm{l}$. The concentration of miR-23b-3p mimic and its negative control used for transfection was $50 \mathrm{nM}$, and that of the inhibitor and its negative control used for transfection was $100 \mathrm{nM}$.

Reverse transcription-quantitative-polymerase chain reaction (RT-qPCR). RT-qPCR was used to detect gene expression levels of hBMSCs at $0,3,7$ and 14 days after osteogenic induction or $72 \mathrm{~h}$ after transfection at $37^{\circ} \mathrm{C}$. Total RNA was extracted by TRIzol (Invitrogen; Thermo Fisher Scientific, Inc.) according to the manufacturer's instructions. RNA samples were reversely transcribed with a commercial reverse transcription kit (Takara Bio, Inc.; cat. no. RR047A) according to the manufacturer's instructions. RT-qPCR was conducted using SYBR Green (Takara Bio, Inc.; cat. no. RR420A) and the ABI 7500 sequence detection system (Applied Biosystems; Thermo Fisher Scientific, Inc.). RT-qPCR thermocycling conditions (40 cycles) were as follows: $95^{\circ} \mathrm{C}$ for $15 \mathrm{sec}$ and $60^{\circ} \mathrm{C}$ for $60 \mathrm{sec}$. All results were normalized to the expression levels of $\beta$-actin or U6 controls and quantified by the comparative $\left(2^{-\Delta \Delta \mathrm{Cq})}\right.$ method (16). The primer sequences used were as follows: RP1184C13.1 forward, 5'-ACAACGTTCTGCGGGGG-3' and reverse, 5'-ATCGCCCTGGGACCGC-3'; $\beta$-actin forward, 5'-CCATGTACGTTGCTATCCAG-3' and reverse, 5'-CTT CATGAGGTAGTCAGTCAG-3'; miR-23b-3p forward, 5'-CGCATCACATTGCCAGGG-3' and reverse, 5'-GTGCAG GGTCCGAGGT-3'; U6 forward, 5'-CTCGCTTCGGCAGCA CA-3' and reverse, 5'-AACGCTTCACGAATTTGCGT-3'.

Western blot analysis. The transfected cells were inoculated into a 6-well plate containing $2 \mathrm{ml}$ of medium and cultured at $37^{\circ} \mathrm{C}$ for $72 \mathrm{~h}$ before the collection. The cells were lysed with radioimmunoprecipitation assay buffer (Nanjing KeyGen BioTECH Co., Ltd.) on ice and centrifuged at 13,000 x g at $4^{\circ} \mathrm{C}$ for $10 \mathrm{~min}$ before the supernatant was collected. The protein concentration was determined by a BCA protein assay kit (Nanjing KeyGen BioTECH Co., Ltd.). After heating at $100^{\circ} \mathrm{C}$ to denature the protein, protein samples $(30 \mu \mathrm{g}$ in each lane) were separated by $10 \%$ SDS-PAGE and transferred onto PVDF membranes (EMD Millipore). The membranes were blocked with $5 \%$ skimmed milk in Tris-buffered saline with $0.2 \%$ Tween-20 (TBST) buffer at room temperature for $1 \mathrm{~h}$. The membranes were washed three times with TBST buffer and incubated with the following antibodies at $4{ }^{\circ} \mathrm{C}$ overnight: runt-related transcription factor 2 (RUNX2; 1:1,000; cat. no. 12556) and $\beta$-actin (1:1,000; cat. no. 4970; both form Cell Signaling Technology, Inc.). The membranes were subsequently incubated with goat anti-rabbit $\operatorname{IgG}(\mathrm{H}+\mathrm{L})$ horseradish peroxidase (HRP)-conjugated secondary antibody (1:5,000; product no. 7074S; Cell Signaling Technology, Inc.) at room temperature for $2 \mathrm{~h}$. Lastly, protein expression was detected 
A

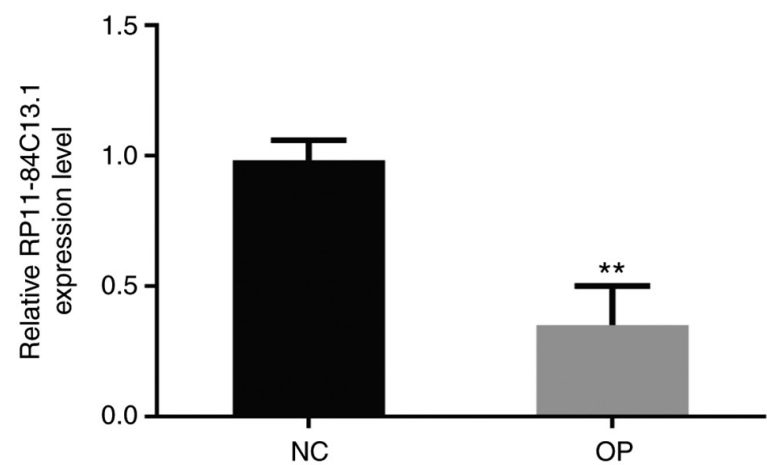

$\mathrm{B}$

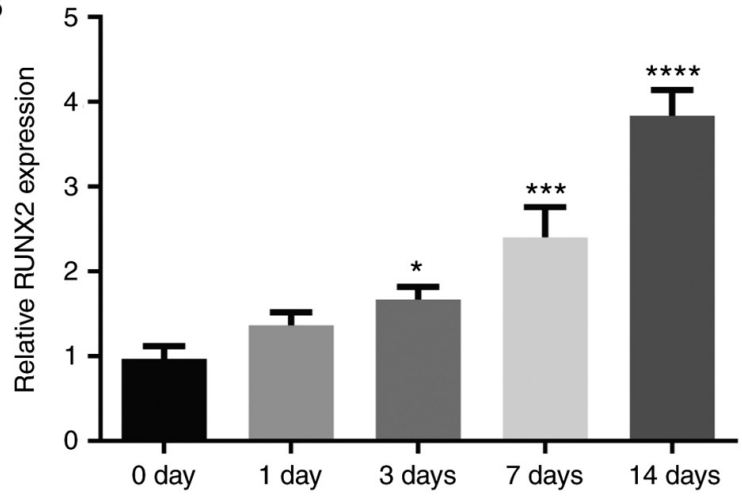

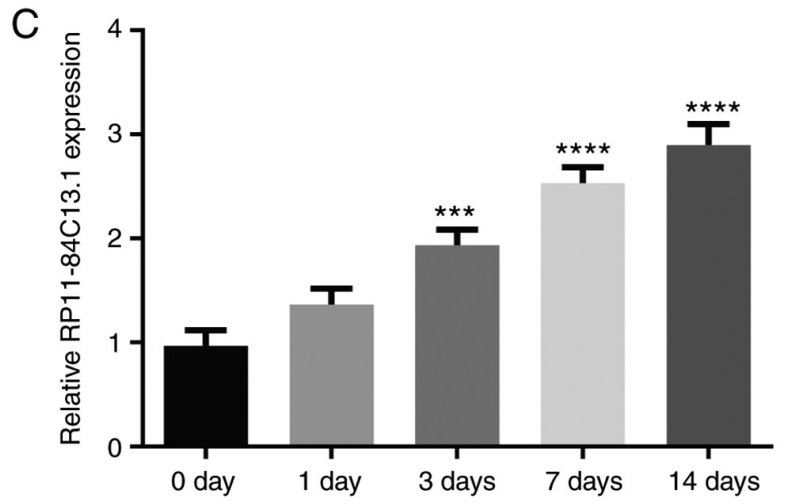

Figure 1. Expression of long non-coding RNA RP11-84C13.1 in osteoporosis patients and hBMSCs. (A) The expression of RP11-84C13.1 was lower in the serum of patients with osteoporosis than the serum from healthy control patients. (B and C) RUNX2 and RP11-84C13.1 expression in hBMSCs was upregulated during osteogenic differentiation. ${ }^{*} \mathrm{P}<0.05,{ }^{* * *} \mathrm{P}<0.001$ and ${ }^{* * * *} \mathrm{P}<0.0001$ vs. the 0 day group; ${ }^{* *} \mathrm{P}<0.01$ vs. the NC group. hBMSCs, human bone marrow stromal cells; RUNX2, runt-related transcription factor 2; NC, normal healthy control; OP, osteoporosis patients.

by chemiluminescent HRP substrate (cat. no. 32132; Thermo Fisher Scientific, Inc.). The signal intensity of primary antibody binding was analyzed using Quantity One software v4.62 (Bio-Rad Laboratories, Inc.).

Bioinformatics analysis. Potential miR-23b-3p binding sites in RP11-84C13.1 were predicted using RNAhybrid (http://bibiserv2.cebitec.uni-bielefeld.de/rnahybrid). Potential miR-23b-3p binding sites in RUNX2 were predicted using TargetScan Human v7.2 (http://www.targetscan.org/).

Dual-luciferase reporter gene assay. Wild-type (wt) RP11-84C13.1, mutant (mut, only the putative miR-23b-3p binding sites were mutated) RP11-84C13.1, wt RUNX2 and mut RUNX2 sequences were constructed into the psiCHECK-2 vector (Promega Corporation). Lipofectamine ${ }^{\mathrm{TM}}$ 2000 (Invitrogen; Thermo Fisher Scientific, Inc.) was used to co-transfect 293T cells (American Type Culture Collection) with the aforementioned plasmids and either miRNA-23b-3p mimic or mimic-NC. Luciferase activity was continuously analyzed by the dual-luciferase assay system (Promega Corporation) $48 \mathrm{~h}$ after transfection. Luciferase data were expressed as a ratio of Renilla luciferase activity to firefly luciferase activity to normalize transfection variability between samples and experiments were repeated in triplicate using independent samples.

Alizarin Red staining. After transfection at $37^{\circ} \mathrm{C}$ for $6 \mathrm{~h}$, hBMSCs were cultured in osteogenic differentiation induction medium for two weeks. The cells were washed with phosphate-buffered saline (PBS) three times and fixed in chilled $70 \%$ ethanol for $1 \mathrm{~h}$. They were then stained with $1 \%$ Alizarin Red (Sigma-Aldrich; Merck $\mathrm{KGaA}$ ) at room temperature for 10-15 min. After rinsing with $\mathrm{ddH}_{2} \mathrm{O}$ to remove the dye, the orange red deposition was quantified using an inverted fluorescence microscope (Olympus Corporation) at a magnification of $x 4$.

Statistical analysis. All experiments were repeated at least three times. Alizarin Red staining results were quantified by ImageJ v1.8.0 (National Institutes of Health). All data are presented as the means \pm standard deviation (SD) and analyzed using SPSS 24.0 software (IBM Corp.). One-way ANOVA, followed by Tukey's post hoc test, were employed to analyze differences among groups. All statistical analyses were calculated using GraphPad Prism 8 (GraphPad Software, Inc.). $\mathrm{P}<0.05$ was considered to indicate a statistically significant difference.

\section{Results}

RP11-84C13.1 expression in osteoporosis patients and hBMSCs. The serum levels of RP11-84C13.1 in osteoporosis patients and normal healthy control patients were detected by RT-qPCR. As revealed in Fig. 1A, RP11-84C13.1 mRNA expression levels in OP were significantly lower than those in NC. To determine whether RP11-84C13.1 participated in the process of osteogenic differentiation, femoral head tissue was 

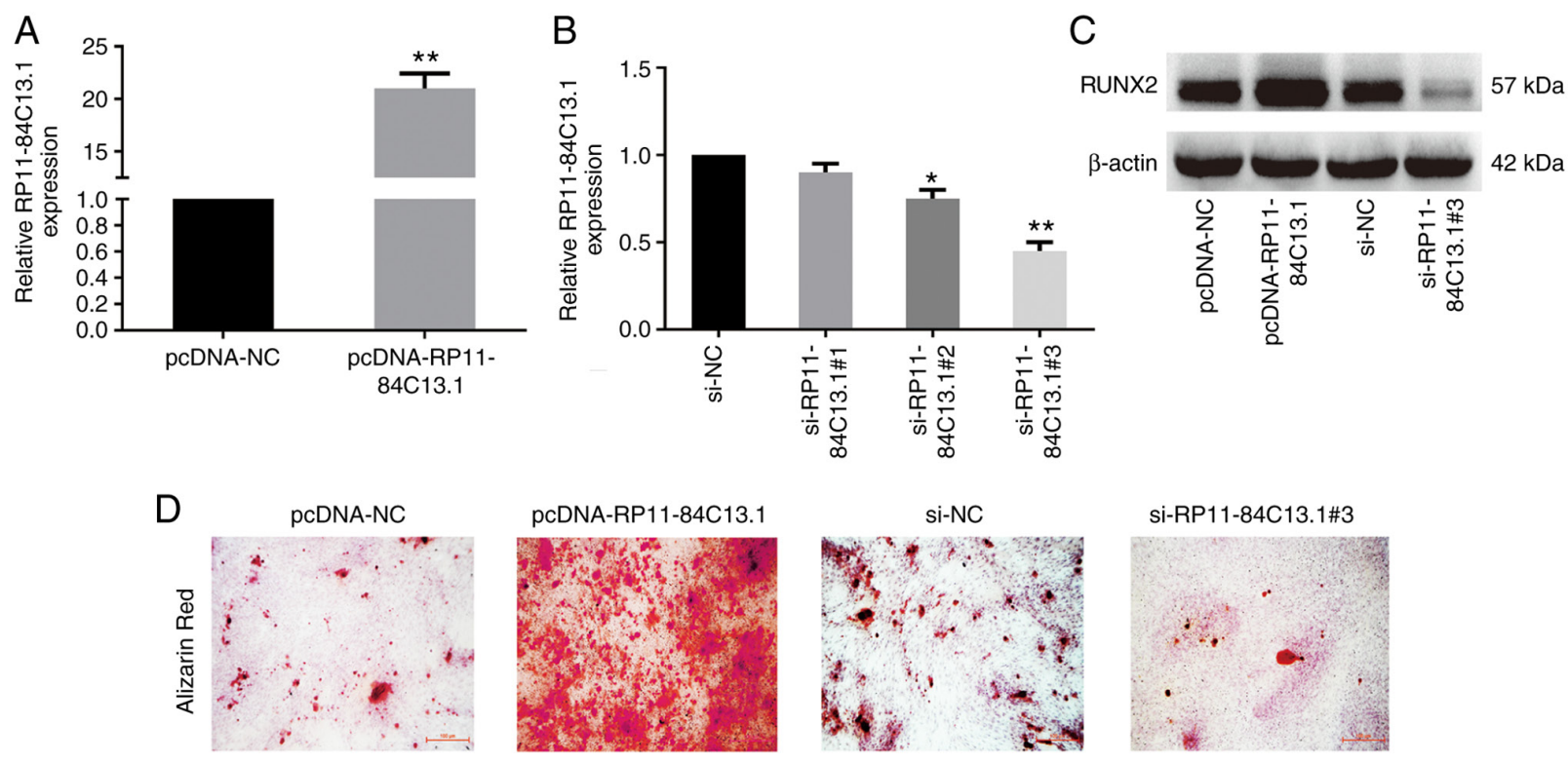

E

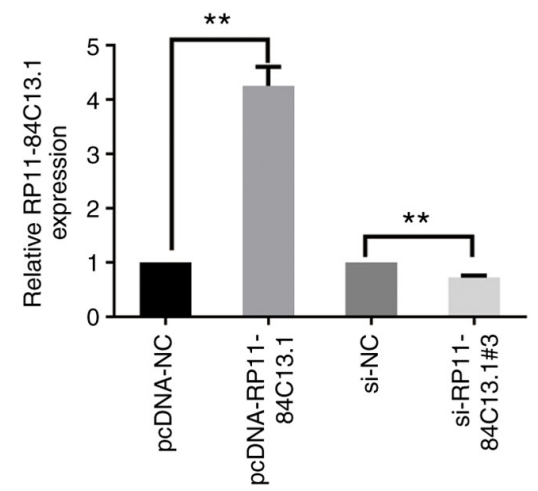

F

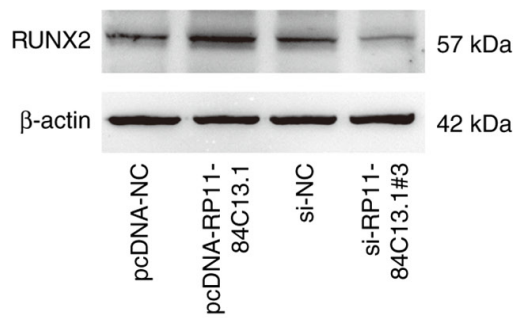

Figure 2.RP11-84C13.1 promotes the osteogenic differentiation of hBMSCs.(A) Following RP11-84C13.1 plasmid transfection for 72h, RP11-84C13.1 expression in hBMSCs was significantly increased. (B) Following transfection with si-RP11-84C13.1 for 72 h, RP11-84C13.1 expression was lowest with si-RP11-84C13.1\#3. (C) After transfection of pcDNA-RP11-84C13.1 and si-RP11-84C13.1\#3 for $72 \mathrm{~h}, \mathrm{RUNX} 2$ protein levels were increased in RP11-84C13.1-overexpressing hBMSCs and decreased in si-RP11-84C13.1\#3 hBMSCs. (D) Alizarin Red staining was decreased in cells following RP11-84C13.1 inhibition but increased in cells with overexpressed RP11-84C13.1. Scale bars, $100 \mu \mathrm{m}$. (E) RP11-84C13.1 levels were increased in RP11-84C13.1-overexpressing hBMSCs and decreased in si-RP11-84C13.1\#3 hBMSCs 14 days after differentiation. (F) After transfection of pcDNA-RP11-84C13.1 and si-RP11-84C13.1\#3, RUNX2 protein levels were increased in RP11-84C13.1-overexpressing hBMSCs and decreased in si-RP11-84C13.1\#3 hBMSCs 14 days after differentiation. " $\mathrm{P}<0.05$ vs. the si-NC group and ${ }^{* *} \mathrm{P}<0.01$ vs. the si-NC group or pcDNA-NC group. hBMSCs, human bone marrow stromal cells; si-, small interfering; RUNX2, runt-related transcription factor $2 \mathrm{NC}$, negative control.

extracted from discarded hBMSCs. The expression of RUNX2 and lncRNA RP11-84C13.1 at 0, 1, 3, 7 and 14 days after osteogenic induction was significantly increased (Fig. 1B and C). These results indicated that RP11-84C13.1 may be involved in osteoporosis progression.

RP11-84C13.1 promotes osteogenic differentiation of hBMSCs. To explore the biological function of RP11-84C13.1 on the osteogenic differentiation of hBMSCs, the transfection efficacy of pcDNA-RP11-84C13.1 and si-RP11-84C13.1 was verified by RT-qPCR. As revealed in Fig. 2A, transfection of pcDNA-RP11-84C13.1 upregulated RP11-84C13.1. Following si-RP11-84C13.1 transfection, RP11-84C13.1 expression was significantly downregulated, and the most significant decrease was induced by si-RP11-84C13.1\#3 (Fig. 2B). Therefore, si-RP11-84C13.1\#3 was selected for the remaining experiments. It was revealed that RUNX2 protein levels were increased in RP11-84C13.1-overexpressing hBMSCs and decreased in si-RP11-84C13.1\#3 hBMSCs (Fig. 2C). Alizarin
Red staining revealed that RP11-84C13.1 knockdown attenuated osteogenic differentiation of hBMSCs (Fig. 2D). To identify if transfections of pcDNA-RP11-84C13.1 and si-RP11-84C13.1\#3 retained their primary effects after 14 days, RT-qPCR and western blot experiments were performed on the differentiated hBMSCs 14 days after induction. RT-qPCR revealed that RP11-84C13.1 levels were increased in RP11-84C13.1-overexpressing hBMSCs and decreased in si-RP11-84C13.1\#3 hBMSCs 14 days after differentiation (Fig. 2E). Western blotting revealed that RUNX2 protein levels were increased in RP11-84C13.1-overexpressing hBMSCs and decreased in si-RP11-84C13.1\#3 hBMSCs 14 days after differentiation (Fig. 2F). Therefore, it was concluded that RP11-84C13.1 promoted the osteogenic differentiation of hBMSCs.

RP11-84C13.1 acts as a sponge of miR-23b-3p. Using bioinformatics analysis, miRNA-23b-3p binding sites in the promoter region of RP11-84C13.1 were predicted (Fig. 3A). 
A

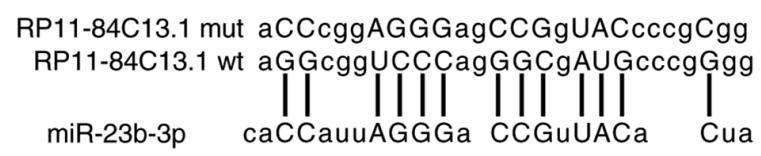

$\mathrm{C}$

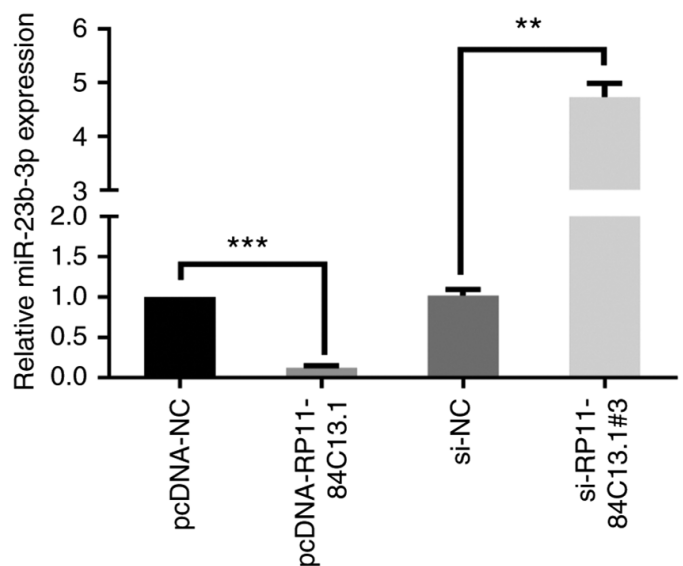

B

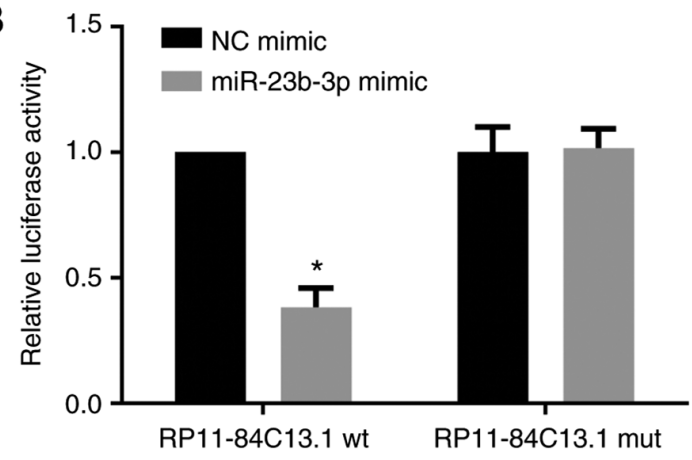

$\mathrm{D}$

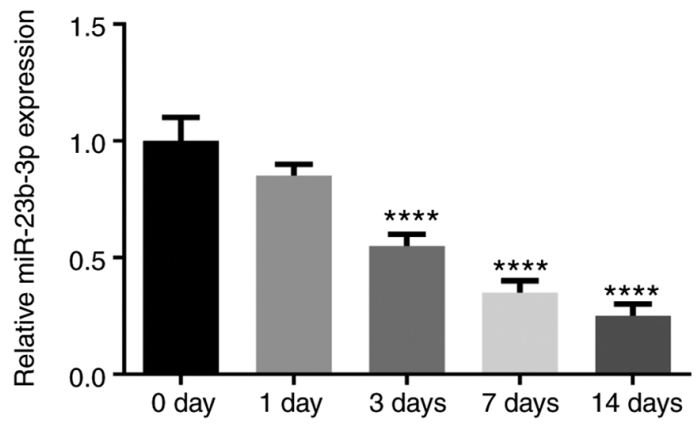

Figure 3. RP11-84C13.1 acts as a sponge of miR-23b-3p. (A) Bioinformatics analysis predicted a binding relationship between RP11-84C13.1 and miR-23b-3p. (B) Dual-luciferase reporter gene assay revealed that miR-23b-3p could bind to wt-RP11-84C13.1. (C) After transfection of pcDNA-RP11-84C13.1 and si-RP11-84C13.1\#3 for $72 \mathrm{~h}$, miR-23b-3p levels were decreased in RP11-84C13.1-overexpressing hBMSCs and increased in si-RP11-84C13.1\#3 hBMSCs. (D) The expression of miRNA-23b-3p in hBMSCs was downregulated during osteogenic differentiation. ${ }^{*} \mathrm{P}<0.05$ vs. the RP11-84C13.1 wt-NC mimic group; ${ }^{* *} \mathrm{P}<0.01$ vs. the si-NC group; ${ }^{* * *} \mathrm{P}<0.001$ vs. the pcDNA-NC group; and ${ }^{* * * * *} \mathrm{P}<0.0001$ vs. the 0 day group. miR, microRNA; wt, wild-type; mut, mutant; si-, small interfering; hBMSCs, human bone marrow stromal cells; NC, negative control.

Dual-luciferase reporter gene assays revealed luciferase fluorescence was quenched in cells co-transfected with miRNA-23b-3p mimic and wt RP11-84C13.1 (Fig. 3B). After overexpression of RP11-84C13.1, the level of miRNA-23b-3p was downregulated, while knockdown of RP11-84C13.1 reversed this downregulation (Fig. 3C). Next, miRNA-23b-3p expression at $0,1,3,7$ and 14 days after osteogenic induction was assessed and a gradual decrease during osteogenic differentiation was observed (Fig. 3D). In conclusion, these results indicated that miRNA-23b-3p could function as a molecular target of RP11-84C13.1 in hBMSCs.

miR-23b-3p inhibits osteogenic differentiation of hBMSCs. Based on the predicted results from TargetScan, the binding sites of miRNA-23b-3p with RUNX2 were identified (Fig. 4A). Dual-luciferase reporter gene assay results indicated that miRNA-23b-3p overexpression significantly decreased the relative luciferase activity of RUNX2-wt, indicating that miRNA-23b-3p directly bound to the 3'UTR of RUNX2 (Fig. 4B). Next, following the transfection of miRNA-23b-3p mimics and an miR-23b-3p inhibitor for $72 \mathrm{~h}$, miR-23b-3p levels were increased in miR-23b-3p-overexpressing hBMSCs and decreased in miR-23b-3p inhibitor hBMSCs, which confirmed the transfection efficiency (Fig. 4C). Furthermore, RUNX2 protein levels were decreased by miRNA-23b-3p mimics and increased by the miRNA-23b-3p inhibitor
(Fig. 4D). Alizarin Red staining revealed elevated calcified nodules in the osteoblasts treated with miRNA-23b-3p inhibitor (Fig. 4E). To identify if transfections of miR-23b-3p mimics and miR-23b-3p inhibitor retained their primary effects after 14 days, RT-qPCR and western blot experiments were performed on the differentiated hBMSCs 14 days after induction. RT-qPCR revealed that miR-23b-3p levels were increased in miR-23b-3p-overexpressing hBMSCs and decreased in miR-23b-3p inhibitor hBMSCs 14 days after differentiation (Fig. 4F). Meanwhile, after transfection of miR-23b-3p mimics and miR-23b-3p inhibitor, RUNX2 protein levels were decreased in miR-23b-3p-overexpressing hBMSCs and increased in miR-23b-3p inhibitor hBMSCs 14 days after differentiation (Fig. 4G). It was concluded that miR-23b-3p inhibited the osteogenic differentiation of hBMSCs.

RP11-84C13.1 regulates osteogenic differentiation of $h B M S C s$ by inhibiting the expression of miR-23b-3p. Since RP11-84C13.1 inhibited miRNA-23b-3p and miRNA-23b-3p directly targeted RUNX2 in hBMSCs, it was further explored whether RP11-84C13.1 could upregulate the expression of RUNX2 by adsorbing miRNA-23b-3p. Our results revealed that upregulated RP11-84C13.1 significantly increased the expression of RUNX2 in hBMSCs, and co-transfection of miRNA-23b-3p mimics partially reversed this increase (Fig. 5A). Alizarin Red staining further revealed that 
A RUNX2 mut aguucauccaggcacUUACACUA

RUNX2 wt aguucauccaggcacAAUGUGAU

miR-23b-3p caccauuagggaccgUUACACUA

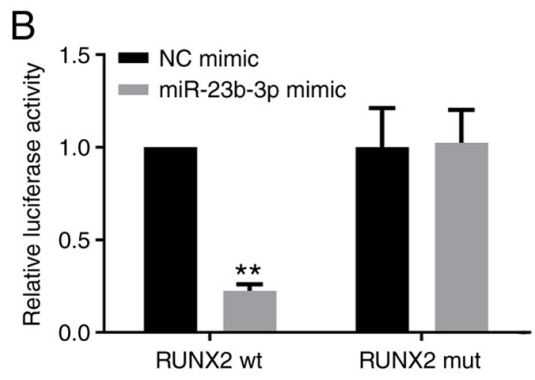

C

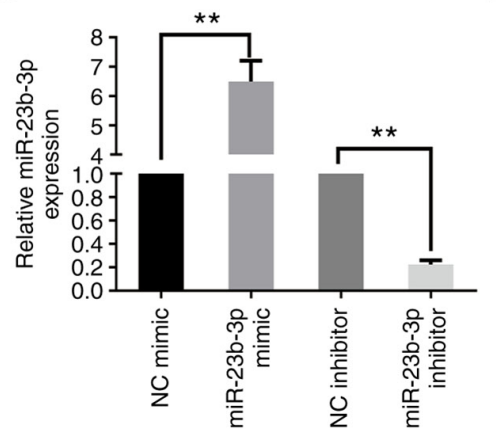

D

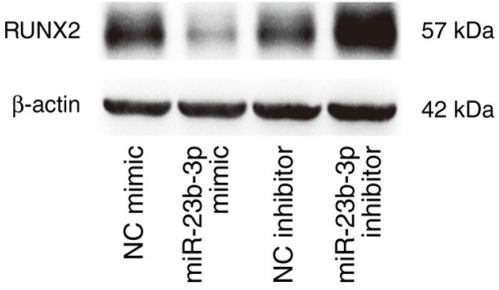

E

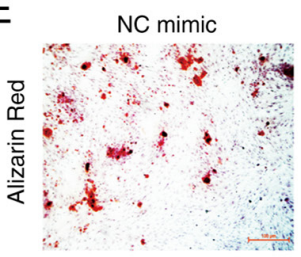

$\mathrm{F}$

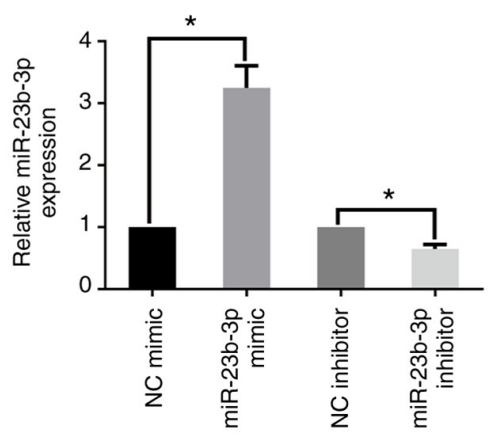

miR-23b-3p mimic
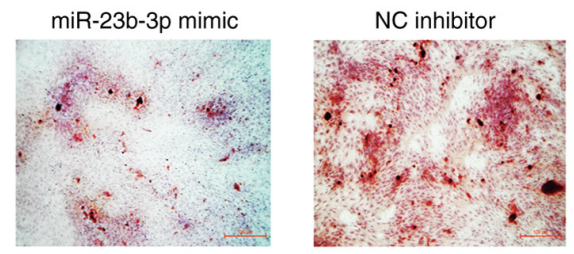

G
miR-23b-3p inhibitor

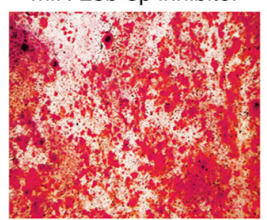

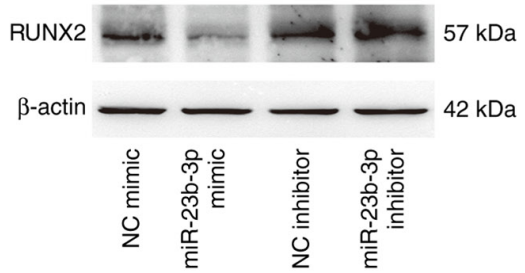

Figure 4. miR-23b-3p inhibits the osteogenic differentiation of hBMSCs. (A) Bioinformatics analysis predicted a binding relationship between miR-23b-3p and RUNX2. (B) Dual-luciferase reporter gene assays revealing that miR-23b-3p could bind to RUNX2 wt. (C) After transfection of miR-23b-3p mimics and a miR-23b-3p inhibitor for $72 \mathrm{~h}$, miR-23b-3p levels were increased in miR-23b-3p-overexpressing hBMSCs and decreased in miR-23b-3p inhibitor hBMSCs. (D) After transfection of miR-23b-3p mimics and a miR-23b-3p inhibitor for $72 \mathrm{~h}$, RUNX2 protein levels were decreased in miR-23b-3p-overexpressing hBMSCs and increased in miR-23b-3p inhibitor hBMSCs. (E) Alizarin Red staining was decreased in cells following miR-23b-3p-overexpression but increased in cells exposed to a miR-23b-3p inhibitor. Scale bars, $100 \mu \mathrm{m}$. (F) miR-23b-3p levels were increased in miR-23b-3p-overexpressing hBMSCs and decreased in miR-23b-3p inhibitor hBMSCs 14 days after differentiation. (G) After transfection of miR-23b-3p mimics and a miR-23b-3p inhibitor, RUNX2 protein levels were decreased in miR-23b-3p-overexpressing hBMSCs and increased in miR-23b-3p inhibitor hBMSCs 14 days after differentiation. ${ }^{*} \mathrm{P}<0.05$ and ${ }^{* *} \mathrm{P}<0.01$ vs. the $\mathrm{NC}$ mimic or $\mathrm{NC}$ inhibitor group. ${ }^{* *} \mathrm{P}<0.01$ vs. the RUNX2 wt-NC mimic group. miR, microRNA; hBMSCs, human bone marrow stromal cells; RUNX2, runt-related transcription factor 2; wt, wild-type; mut, mutant; NC, negative control.

overexpression of miRNA-23b-3p rescued the maximization nodule formation in hBMSCs (Fig. 5B). Moreover, after transfection of RP11-84C13.1 plasmid, RUNX2 protein levels were increased in RP11-84C13.1-overexpressing hBMSCs 14 days after differentiation. Co-transfection of miRNA-23b-3p mimics partially reversed this increase (Fig. 5C). These results indicated that $\mathrm{RP} 11-84 \mathrm{C} 13.1$ regulated osteogenic differentiation of hBMSCs by inhibiting the expression of miR-23b-3p.

\section{Discussion}

Osteoporosis is a common and costly bone metabolic disorder, common in elderly and postmenopausal women, that results in bone loss and fracture risk (17). The major reason for the failure to maintain bone homeostasis is an imbalance between bone resorption and bone formation caused by decreased osteoblast activity $(18,19)$. To further understand the pathogenesis of osteoporosis, the transcription network and signaling pathways at the genetic level were investigated to identify novel targets for curative osteoporosis treatment.

Recently, emerging research has illustrated the significance of lncRNAs as important regulators in osteoclastogenesis and osteoblast differentiation. They can play a vital role by regulating cell structural integrity, controlling subcellular localization and inducing epigenetic modification (20-22). For example, lncRNA SNHG1 has been revealed to attenuate osteogenic differentiation via the miR-101/DKK1 axis in hBMSCs (23). LncRNA BCAR4 is involved in the osteogenic differentiation of BMSCs, and silencing BCAR4 can alleviate 
A

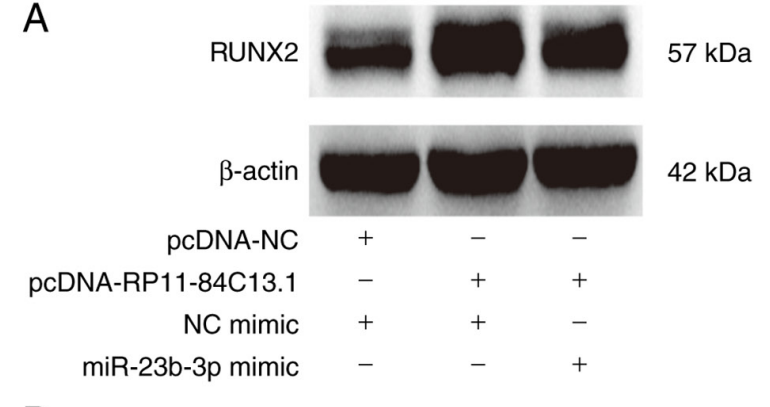

B
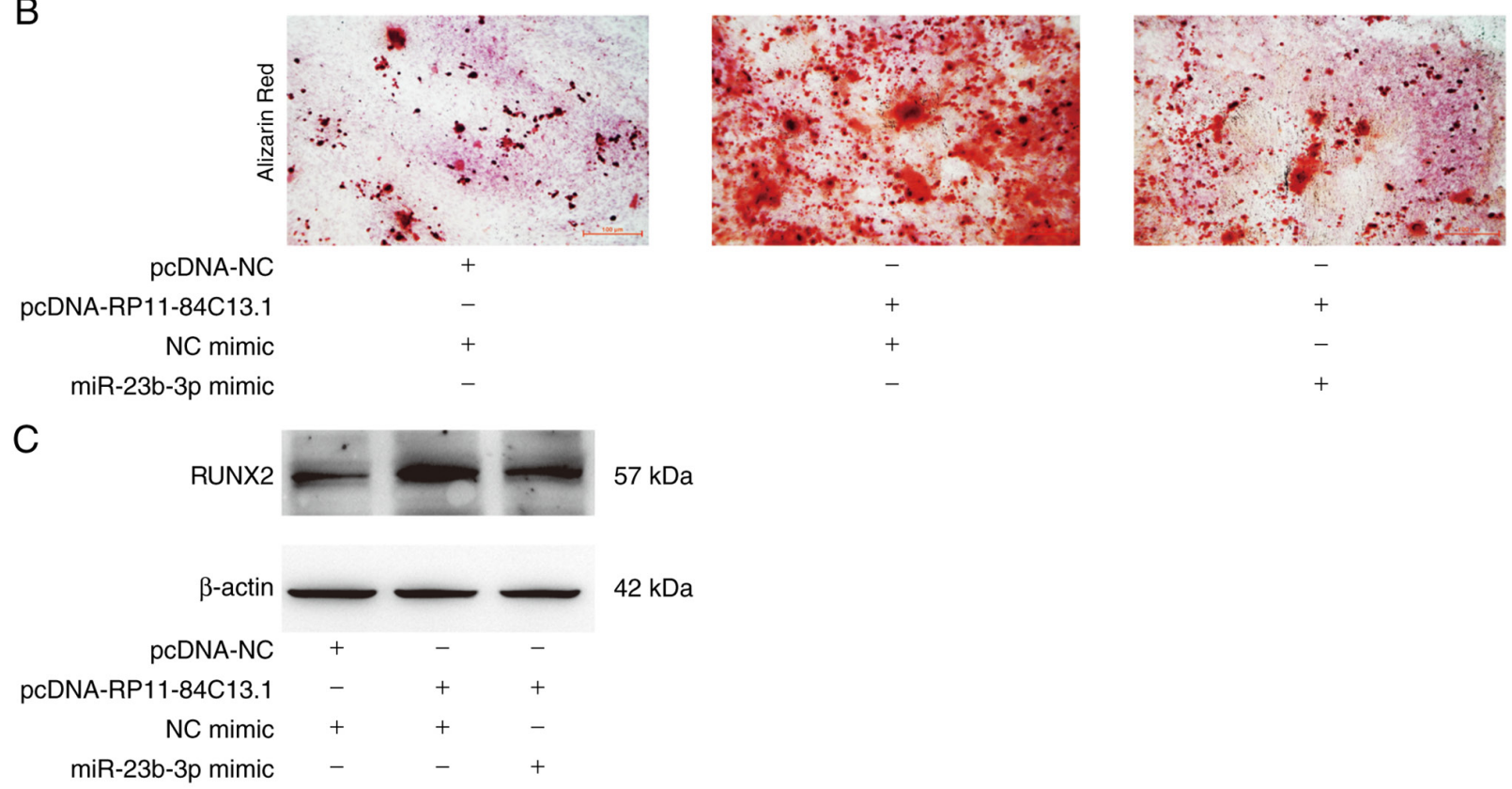

Figure 5. RP11-84C13.1 regulates osteogenic differentiation of hBMSCs by inhibiting the expression of miR-23b-3p. (A) After transfection of pcDNA-RP11-84C13.1 for $72 \mathrm{~h}$, overexpression of miR-23b-3p decreased RUNX2 expression. (B) Overexpression of miR-23b-3p reversed the Alizarin Red staining after RP11-84C13.1 overexpression. Scale bars, $100 \mu \mathrm{m}$. (C) After transfection of pcDNA-RP11-84C13.1, RUNX2 protein levels were decreased in miR-23b-3p-overexpressing hBMSCs 14 days after differentiation. hBMSCs, human bone marrow stromal cells; miR, microRNA; RUNX2, runt-related transcription factor 2 ; NC, negative control

the deterioration of osteoporosis (24). LncRNA ODSM inhibits osteoblast apoptosis and promotes osteoblast mineralization in vitro (25). Based on clinical research, a novel lncRNA, RP11-84C13.1, was identified in osteoporosis patients (11). Firstly, our study focused on the expression of RP11-84C13.1 in osteoporosis and it was revealed that RP11-84C13.1 was downregulated in the progression of osteoporosis. Then, to further understand the function of RP11-84C13.1, overexpression and knockdown experiments revealed that RP11-84C13.1 could promote the osteogenic differentiation of hBMSCs.

Although the role of RP11-84C13.1 in osteoporosis has been preliminarily studied, our current knowledge of its basic pathophysiological mechanism remains unclear. To date, multiple RNA transcripts, such as lncRNAs and circular RNAs, have been revealed to function as competitive endogenous RNAs by miRNA binding $(26,27)$. Wang et al $(28)$ revealed that lncRNA KCNQ1OT1 promoted BMP2 expression to regulate osteogenic differentiation by sponging miRNA-214. Ji et al (29)reported that hsa_circ_0026827 promoted osteoblast differentiation of human dental pulp stem cells through the beclin1 and RUNX1 signaling pathways by sponging miR-188-3p. Using bioinformatics analyses, it was predicted that miR-23b-3p targeted RP11-84C13.1. miR-23b-3p participates in multifactorial disease. miR-23b-3p reduced the proliferation, migration and invasion of cervical cancer cell lines via reduced c-Met expression (30). miR-23b-3p was revealed to have a higher clinical diagnostic efficacy and may be a potential biomarker for early diagnosis of non-small cell lung cancer (31). miR-23 also decreased osteogenic differentiation of hBMSCs through the MEF2C/MAPK signaling pathway (32). Additionally, miR-23b-3p was negatively regulated by RP11-84C13.1. Finally, miR-23b-3p was predicted to directly target RUNX2, and its overexpression could partially reverse the regulatory effects of RP11-84C13.1 on osteogenesis of hBMSCs.

RUNX2, located on chromosome $6 \mathrm{p} 21$, is one of the most critical transcription factors involved in osteoporosis, and its deficiency inhibits osteoblast differentiation, contributing to the development of diseases $(33,34)$, including numerous bone-related diseases (35-37). The results of the present study determined that expression of RP11-84C13.1 was positively associated to RUNX2 and promoted osteogenic differentiation of hBMSCs. miR-23b-3p expression was negatively associated to RUNX2 and inhibited osteogenic differentiation of hBMSCs. Collectively, these data validated that RP11-84C13.1 regulated RUNX2 by competing for miR-23b-3p binding in osteoporosis. siRNAs have been used 
to study a variety of diseases, including osteoporosis. By Alizarin Red staining, it was revealed that osteogenic differentiation of hBMSCs was induced by transfection of si-STAT5a for 14 days (38); osteogenic differentiation of mBMSCs was inhibited by transfection of si-KLF5 for 14 days (39); the osteogenic ability of CON-ASCs was decreased by transfection of si-Jkamp for 14 days (40); osteogenic differentiation of hJBMMSCs was reduced by transfection of si-SEMA3A for 14 days (37). In general, siRNAs have a short role time in cells, which may be attributed to the gradual dilution of siRNAs after transfection in cell replication, so that the gene silencing effect gradually disappears. In the present study of osteoblast differentiation, the serum of cell cultures was reduced from 10 to $1 \%$ to avoid possible proliferation and differentiation, which may be the possible mechanism by which the siRNA remained effective at 14 days of osteoblast differentiation.

However, the present study still has some limitations. Firstly, these results have not been confirmed in vivo. Therefore, experiments will be conducted to verify the results in animal experiments. Secondly, the epigenetic modification of RP11-84C13.1 was not investigated. Although the aforementioned findings and a previous study have revealed that RP11-84C13.1 is downregulated in osteoporosis (11), the mechanism for RP11-84C13.1 dysregulation in osteoporosis remains unclear. A few studies have revealed that epigenetic modification can regulate lncRNA transcription. For example, an osteoporosis risk SNP at 1p36.12 acts as an allele-specific enhancer to modulate LINC00339 expression via long-range loop formation (41). LncRNA GAS5 has been revealed to inhibit progression of colorectal cancer by interacting with and triggering YAP phosphorylation and degradation and was negatively regulated by the m6A reader YTHDF3 (42). DNMT1 has been revealed to control the IncRNA H19/ERK signaling pathway in hepatic stellate cell activation and fibrosis (43). TEAD4-modulated lncRNA MNX1-AS1 contributed to gastric cancer progression partly by suppressing BTG2 and activating BCL2 (44). Thus, the exact regulatory mechanisms of RP11-84C13.1 downregulation in osteoporosis still require further exploration.

In summary, it was determined for the first time that RP11-84C13.1 induced osteogenesis of hBMSCs. More specifically, RP11-84C13.1 inhibited the expression of miR-23b-3p by competitive binding with miR-23b-3p and promoted the expression of RUNX2 to induce the osteogenic differentiation of hBMSCs.

\section{Acknowledgements}

Not applicable.

\section{Funding}

The present study was supported by the National Natural Science Foundation of China for Youth (grant no. 81700776).

\section{Availability of data and materials}

All data generated or analyzed during the present study are included in this published article.

\section{Authors' contributions}

HY and LC contributed to the conception and design of the study. HY, JT, XL, WH, and LC contributed to the conduction of the study and data collection. HY, YL, and LC contributed to data analysis. HY and LC wrote the manuscript. All authors read and approved the final manuscript. HY and LC confirm the authenticity of all the raw data.

\section{Ethics approval and consent to participate}

This study was approved by the Medical Ethics Committee of Huai'an Second People's Hospital (Huai'an, China), and patient consent was obtained. Written informed consent was obtained from all the participants.

\section{Patient consent for publication}

Not applicable.

\section{Competing interests}

The authors declare that they have no competing interests.

\section{References}

1. Langdahl B, Ferrari S and Dempster DW: Bone modeling and remodeling: Potential as therapeutic targets for the treatment of osteoporosis. Ther Adv Musculoskelet Dis 8: 225-235, 2016.

2. Zhou S, Huang G and Chen G: Synthesis and biological activities of drugs for the treatment of osteoporosis. Eur J Med Chem 197: $112313,2020$.

3. Feehan J, Al Saedi A and Duque G: Targeting fundamental aging mechanisms to treat osteoporosis. Expert Opin Ther Targets 23: 1031-1039, 2019

4. Mendoza FA, Le Roux M and Ahmed I: Primary osteoporosis in men: An unmet medical need. Fertil Steril 112: 791-798, 2019.

5. Zhu Y, Huang Z, Wang Y, Xu W, Chen H, Xu J, Luo S, Zhang Y, Zhao D and Hu J: The efficacy and safety of denosumab in postmenopausal women with osteoporosis previously treated with bisphosphonates: A review. J Orthop Translat 22: 7-13, 2020.

6. Cao DW, Liu MM, Duan R, Tao YF, Zhou JS, Fang WR, Zhu JR, Niu L and Sun JG: The lncRNA Malat1 functions as a ceRNA to contribute to berberine-mediated inhibition of HMGB1 by sponging miR-181c-5p in poststroke inflammation. Acta Pharmacol Sin 41: 22-33, 2020.

7. Huynh NP, Anderson BA, Guilak F and McAlinden A: Emerging roles for long noncoding RNAs in skeletal biology and disease. Connect Tissue Res 58: 116-141, 2017.

8. Del Real A, López-Delgado L, Sañudo C, García-Ibarbia C, Laguna E, Perez-Campo FM, Menéndez G, Alfonso A, Fakkas M, García-Montesinos B, et al: Long noncoding RNAs as bone marrow stem cell regulators in osteoporosis. DNA Cell Biol 39: 1691-1699, 2020.

9. Peng S, Cao L, He S, Zhong Y, Ma H, Zhang Y and Shuai C: An overview of long noncoding RNAs involved in bone regeneration from mesenchymal stem cells. Stem Cells Int 2018: 8273648, 2018.

10. Chen X, Yang L, Ge D, Wang W, Yin Z, Yan J, Cao X, Jiang C, Zheng $\mathrm{S}$ and Liang B: Long non-coding RNA XIST promotes osteoporosis through inhibiting bone marrow mesenchymal stem cell differentiation. Exp Ther Med 17: 803-811, 2019.

11. Centofanti F, Santoro M, Marini M, Visconti VV, Rinaldi AM, Celi M, D'Arcangelo G, Novelli G, Orlandi G, Tancredi V, et al: Identification of aberrantly-expressed long non-coding RNAs in osteoblastic cells from osteoporotic patients. Biomedicines 8: 65, 2020.

12. Ge DW, Wang WW, Chen HT, Yang L and Cao XJ: Functions of microRNAs in osteoporosis. Eur Rev Med Pharmacol Sci 21: 4784-4789, 2017.

13. Sun M, Zhou X, Chen L, Huang S, Leung V, Wu N, Pan H, Zhen W, Lu W and Peng S: The regulatory roles of MicroRNAs in bone remodeling and perspectives as biomarkers in osteoporosis. Biomed Res Int 2016: 1652417, 2016. 
14. Zhang M, Cheng L and Zhang Y: Characterization of dysregulated lncRNA-associated ceRNA network reveals novel lncRNAs with ceRNA activity as epigenetic diagnostic biomarkers for osteoporosis risk. Front Cell Dev Biol 8: 184, 2020.

15. Zheng C, Bai C, Sun Q, Zhang F, Yu Q, Zhao X, Kang S, Li J and Jia Y: Long noncoding RNA XIST regulates osteogenic differentiation of human bone marrow mesenchymal stem cells by targeting miR-9-5p. Mech Dev 162: 103612, 2020.

16. Livak KJ and Schmittgen TD: Analysis of relative gene expression data using real-time quantitative PCR and the 2(-Delta Delta C(T)) method. Methods 25: 402-408, 2001.

17. Zhang HL, Du XY and Dong QR: LncRNA XIXT promotes osteogenic differentiation of bone mesenchymal stem cells and alleviates osteoporosis progression by targeting miRNA-30a-5p. Eur Rev Med Pharmacol Sci 23: 8721-879, 2019

18. Wang K, Wang Y, Hu Z, Zhang L, Li G, Dang L, Tan Y, Cao X Shi F, Zhang S and Zhang G: Bone-targeted lncRNA OGRU alleviates unloading-induced bone loss via miR-320-3p/Hoxa10 axis. Cell Death Dis 11: 382, 2020.

19. Zhang Y, Chen B, Li D, Zhou X and Chen Z: LncRNA NEAT1/miR-29b-3p/BMP1 axis promotes osteogenic differentiation in human bone marrow-derived mesenchymal stem cells. Pathol Res Pract 215: 525-531, 2019.

20. Zhang Y, Chen XF, Li J, He F, Li X and Guo Y: lncRNA Neat1 stimulates osteoclastogenesis via sponging miR-7. J Bone Miner Res 35: 1772-1781, 2020.

21. Gao Y, Xiao F, Wang C, Wang C, Cui P, Zhang X and Chen X: Long noncoding RNA MALAT1 promotes osterix expression to regulate osteogenic differentiation by targeting miRNA-143 in human bone marrow-derived mesenchymal stem cells. J Cell Biochem 119: 6986-6996, 2018.

22. Wang CG, Hu YH, Su SL and Zhong D: LncRNA DANCR and miR-320a suppressed osteogenic differentiation in osteoporosis by directly inhibiting the Wnt/ $\beta$-catenin signaling pathway. Exp Mol Med 52: 1310-1325, 2020.

23. Xiang J, Fu HQ, Xu Z, Fan WJ, Liu F and Chen B: LncRNA SNHG1 attenuates osteogenic differentiation via the miR-101/DKK1 axis in bone marrow mesenchymal stem cells. Mol Med Rep 22: 3715-3722, 2020.

24. Li DJ, Liu GQ and Xu XJ: Silence of lncRNA BCAR4 alleviates the deterioration of osteoporosis. Eur Rev Med Pharmacol Sci 24: 5905-5913, 2020.

25. Wang Y, Wang K, Zhang L, Tan Y, Hu Z, Dang L, Zhou H, Li G, Wang $\mathrm{H}$, Zhang $\mathrm{S}$, et al: Targeted overexpression of the long noncoding RNA ODSM can regulate osteoblast function in vitro and in vivo. Cell Death Dis 11: 133, 2020.

26. Chen Q, Wang M and Wu S: The lncRNA MCF2L-AS1 controls osteogenic differentiation by regulating miR-33a. Cell Cycle 19 $1059-1065,2020$

27. Yin Q, Wang J, Fu Q, Gu S and Rui Y: CircRUNX2 through has-miR-203 regulates RUNX2 to prevent osteoporosis. J Cell Mol Med 22: 6112-6121, 2018

28. Wang CG, Liao Z, Xiao H, Liu H, Hu YH, Liao QD and Zhong D: LncRNA KCNQ1OT1 promoted BMP2 expression to regulate osteogenic differentiation by sponging miRNA-214. Exp Mol Pathol 107: 77-84, 2019.

29. Ji F, Zhu L, Pan J, Shen Z, Yang Z, Wang J, Bai X, Lin Y and Tao J: hsa_circ 0026827 promotes osteoblast differentiation of human dental pulp stem cells through the beclin1 and RUNX1 signaling pathways by sponging miR-188-3p. Front Cell Dev Biol 8: 470, 2020.

30. Campos-Viguri GE, Peralta-Zaragoza O, Jiménez-Wences $\mathrm{H}$, Longinos-González AE, Castañón-Sánchez CA, Ramírez-Carrillo M, Camarillo CL, Castañeda-Saucedo E, Jiménez-LópezMA,Martínez-CarrilloDNandFernández-TilapaG: MiR-23b-3p reduces the proliferation, migration and invasion of cervical cancer cell lines via the reduction of c-Met expression. Sci Rep 10: 3256, 2020.
31. Wang J, Xue H, Zhu Z, Gao J, Zhao M and Ma Z: Expression of serum exosomal miR-23b-3p in non-small cell lung cancer and its diagnostic efficacy. Oncol Lett 20: 30, 2020.

32. Jiang K, Teng GD and Chen YQ: MicroRNA-23 suppresses osteogenic differentiation of human bone marrow mesenchymal stem cells by targeting the MEF2C-mediated MAPK signaling pathway. J Gene Med 22: e3216, 2020.

33. Gao M, Sun L, Xu K, Zhang L, Zhang Y, He T, Sun R, Huang H, Zhu J, Zhang Y, et al: Association between low-to-moderate fluoride exposure and bone mineral density in Chinese adults: Non-negligible role of RUNX2 promoter methylation. Ecotoxicol Environ Saf 203: 111031, 2020.

34. Kim WJ, Shin HL, Kim BS, Kim HJ and Ryoo HM RUNX2-modifying enzymes: Therapeutic targets for bone diseases. Exp Mol Med 52: 1178-1184, 2020.

35. Gomathi K, Akshaya N, Srinaath N, Moorthi A and Selvamurugan N: Regulation of Runx 2 by post-translational modifications in osteoblast differentiation. Life Sci 245: 117389, 2020.

36. Narayanan A, Srinaath N, Rohini M and Selvamurugan N: Regulation of Runx 2 by MicroRNAs in osteoblast differentiation. Life Sci 232: 116676, 2019.

37. Lu M, Guo S, Hong F, Zhang Y, Yuan L, Ma C and Ma J: Pax2 is essential for proliferation and osteogenic differentiation of mouse mesenchymal stem cells via Runx2. Exp Cell Res 371: 342-352, 2018

38. Lee KM, Park KH, Hwang JS, Lee M, Yoon DS, Ryu HA, Jung HS, Park KW, Kim J, Park SW, et al: Inhibition of STAT5A promotes osteogenesis by DLX5 regulation. Cell Death Dis 9: 1136,2018

39. Li L, Wang H, Chen X, Li X, Wang G, Jie Z, Zhao X, Sun X, Huang $\mathrm{H}$, Fan $S$, et al: Oxidative stress-induced hypermethylation of KLF5 promoter mediated by DNMT3B impairs osteogenesis by diminishing the interaction with $\beta$-catenin. Antioxid Redox Signal 35: 1-20, 2021.

40. Peng S, Shi S, Tao G, Li Y, Xiao D, Wang L, He Q, Cai X and Xiao J: JKAMP inhibits the osteogenic capacity of adipose-derived stem cells in diabetic osteoporosis by modulating the Wnt signaling pathway through intragenic DNA methylation. Stem Cell Res Ther 12: 120, 2021

41. Chen XF, Zhu DL, Yang M, Hu WX, Duan YY, Lu BJ, Rong Y, Dong SS, Hao RH, Chen JB, et al: An osteoporosis risk SNP at $1 \mathrm{p} 36.12$ acts as an allele-specific enhancer to modulate LINC00339 expression via long-range loop formation. Am J Hum Genet 102: 776-793, 2018.

42. Ni W, Yao S, Zhou Y, Liu Y, Huang P, Zhou A, Liu J, Che L and Li J: Long noncoding RNA GAS5 inhibits progression of colorectal cancer by interacting with and triggering YAP phosphorylation and degradation and is negatively regulated by the $\mathrm{m}^{6} \mathrm{~A}$ reader YTHDF3. Mol Cancer 18: 143, 2019.

43. Yang JJ, She Q, Yang Y, Tao H and Li J: DNMT1 controls LncRNA H19/ERK signal pathway in hepatic stellate cell activation and fibrosis. Toxicol Lett 295: 325-334, 2018.

44. Shuai Y, Ma Z, Liu W, Yu T, Yan C, Jiang H, Tian S, Xu T and Shu Y: TEAD4 modulated LncRNA MNX1-AS1 contributes to gastric cancer progression partly through suppressing BTG2 and activating BCL2. Mol Cancer 19: 6, 2020.

This work is licensed under a Creative Commons Attribution-NonCommercial-NoDerivatives 4.0 International (CC BY-NC-ND 4.0) License. 